\title{
Science, Culture, and Care in Laboratory Animal
}

\section{Research: Interdisciplinary perspectives on the history}

\section{and future of the 3 Rs}

\section{Authors}

Prof. Gail Davies, Professor in Human Geography. Department of Geography, Amory Building, University of Exeter, Rennes Drive, Exeter, EX4 4RJ, UK. +44 (0) 1392 723346, g.f.davies@exeter.ac.uk

Dr Beth Greenhough, Associate Professor in Human Geography. School of Geography and the Environment, Oxford University Centre for the Environment, University of Oxford, South Parks Road, Oxford, OX1 3QY, UK. +44 (0)1865 614924, beth.greenhough@ouce.ox.ac.uk

Dr Pru Hobson-West, Associate Professor in Welfare, Ethics and Society, Faculty of Medicine \& Health Sciences. School of Veterinary Medicine and Science, Vet School Building, Sutton Bonington Campus, Sutton Bonington, Leicestershire, LE12 5RD, UK. +44 (0)115 951 6325, Pru. Hobsonwest@nottingham.ac.uk

Dr Robert G.W. Kirk, Lecturer in the History of Science Technology and Medicine. Centre for the History of Science Technology and Medicine (CHSTM), Faculty of Biology, Medicine and Health, Simon Building 2nd Floor RM 2.71, University of Manchester, Brunswick Street, Manchester, M13 9PL, UK. +44

(0)1612755919, robert.g.kirk@manchester.ac.uk

\section{Keywords}

Laboratory animals, ethics, responsibility, care, sentience, Haraway

\section{Abstract}

The need to refine, replace and reduce the use of animals in laboratories - the 3Rs concept" - strongly influences discussion and regulation of this contested area of technoscience. This special issue looks back to the origins of the 3 Rs concept through five papers that explore how it is enacted and challenged in practice, and that develop critical considerations about its future. Three themes connect the papers in this special issue. These are (1) the multiplicity of roles enacted by those who use and care for animals in research; (2) the distribution of "feelings that matter" across species and spaces of laboratory animal practice; (3) the growing importance of "cultures of care" in animal research. 


\section{Introduction: Response-ability in laboratory animal research}

In 2008 Donna Haraway proposed a way of thinking about ethical relations among humans and animal in bioscience research laboratories; she eschewed guiding principles in favor of attending to entangled subjectivities. Her book opens with a fictional moment when an animal caretaker inserts his arm into a cage, to share the suffering of the guinea pigs housed there, inviting the resident flies to bite his arm. From this, Haraway proposes the potential of the laboratory as a site of ethical encounter among scientists, technicians, and the animals with whom they work. She argues these are not merely places where animals are instrumentalized, though this is an undeniable part of their scientific rationale and organization; they may also be places from which to acknowledge shared suffering and cultivate respect and responsibility. For Haraway, respect is about recognizing both otherness and connection in the moment of "looking back, holding in regard, understanding that meeting the look of the other is a condition of being oneself" $(2008,88)$. Response-ability follows from attending to these entanglements of science and care, human and animal, subject and object: "to be in response is to recognize co-presence in relations of use and therefore to remember that no balance sheet of benefit and cost will suffice" (2008, 76). Here, Haraway is building from, and responding to, the work of Vinciane Despret (2004), who insists on the ethical value of a shared interest in the other. By making the entangled subjectivities of human and animal the focus of her empirical studies of scientific encounters, Despret reveals "that learning how to address the creatures being studied is not the result of scientific theoretical understanding, it is the condition of this understanding" (Despret, 2004, 131). Animal use and animal care are not separated, but connected through capacities to recognize and respond to the suffering of another; what is inadequate in this conceptual formulation are the administrative techniques like cost (or more usually harm)/benefit analysis in accounting for this ethical relation.

Haraway's work has shaped a new set of narratives about animal research, which not only stress the processes of instrumentalizing or calculating within animal research, but also show how "people and animals in labs are both subjects and objects to each other in ongoing intra-action" $(2008,71)$. Her work on respect and responsibility has helped social scientists study how response-abilities are enacted not through "Principles and Ethical Universals but in practices and imaginative politics of the sort that rearticulate [...] the relations of minds and bodies, in this case critters and their lab people and scientific apparatuses" (Haraway 2008, 89). This reformulation provoked debate within critical animal studies (Weisberg 2009) and paved the way for ethnographic accounts of the ethical relations and response-able practices of laboratory animal husbandry and research. These combine earlier work in STS on the material and symbolic transformations of sentient animal bodies into research data (for example, Lynch 1988; Birke et al. 2007) with a formulation of interspecies intra-action, affective encounter, and ethical response-ability (see for example, Greenhough and Roe 2010; Holmberg 2011; Davies 2012a; Friese 2013; Dam and Svendsen 2017). They often look at the wider contexts that engender or hinder ethical ways of 
relating as the growing demands of translational science or increasing positions of scientific precarity place animals and researchers in troublesome relations and positions (Davies 2012b; Johnson 2015; Nelson 2016). Yet, there remain important questions around how far the practices and politics of laboratory animal research, which Haraway's and other work that has followed articulate, connect with other ongoing conversations (particularly within laboratory animal communities) concerning the more bureaucratic aspects of science, culture, and care. Here, a different set of "Rs" circulates, shaping the political and imaginative spaces in which care, respect and responsibility are practiced. These are the $3 \mathrm{Rs}$ of replacement, reduction, and refinement of animal research.

The 3Rs-first systematically articulated in oft-cited but little-read book by Russell and Burch, The Principles of Humane Experimental Technique (1959) - have become fundamental to the definition of humane research and improvement of animal welfare within the international laboratory animal research community. Together, the $3 \mathrm{Rs}$ propose an approach to animal research that prioritizes the replacement of animals with alternative mechanisms, where possible; the reduction of the number of animals required for a given procedure through statistical or other improvements; and the refinement of experimental procedures to minimize suffering and improve animal welfare. The 3Rs have formed the basis for the regulation and professionalization of laboratory animal welfare across policy making, animal care and housing, experimental protocols, and ethical review, especially in Europe and the UK. The 3Rs now provide the core of political commitments to, and social understandings of, ethical animal experimentation; they are embedded in national and EU legislation and international guidelines and are becoming central to the policies of many organizations that fund and conduct animal research. Responsibility was not listed as one of original $3 R s$, but the idea of a $4^{\text {th }} R$ of responsibility underpinning all the others has been proposed several times in the past (Banks, 1995) and does appear in many institutional commitments around animal research ${ }^{1}$ (see also MacLeod and Hartley 2017).

This special issue emerged from an initiative to bring humanities and social sciences work on laboratory animal science and care into dialogue with the policies and practices of animal research. A small grant from the Wellcome Trust brought the authors and other participants together to share previous work and explore the future of the 3 Rs. This special issue is one of the outputs of this initiative. Further outputs included a collaborative agenda-setting article (see Davies et al. 2016), and a larger Wellcome Trust funded grant on The Animal Research Nexus led by Davies, Greenhough, Kirk, Hobson-West, and Roe. The five papers in this issue trace how the $3 R$ s and other related bureaucratic activities have transformed laboratory animal care. They also respond to the challenge at the end of Haraway's chapter, where Sharon Ghamari-Tabrizi probes at the limits of an ethical theory based on co-presence and trans-species relationality, asking: "I still want to know how specifically laboratory experimental practices get done and

\footnotetext{
${ }^{1}$ For example at the Max Planck Society https://www.mpg.de/10973438/4rs (accessed 8/11/2017)
} 
get justified. These details, these mundane practices, are the place where the politics of successor science get worked out" (cited in Haraway 2008, 87).

This special issue returns to questions in STS around laboratory animal care, paying attention to the construction of laboratory animal environments and roles, the distribution of permissions and prohibitions, and the combinations of science and care within experimental practices. This foregrounds how specific "laboratory experimental practices get done and get justified" and demonstrates how "these mundane practices" might shape the contours of a successor science. As the papers demonstrate, the scientific and sociological imaginations, which construct the world of experimental animals, already embody a complex array of ethical propositions and different political positions, whether in setting limits to trading-off harms to animals and human benefits, distributing responsibilities for attending to both animal suffering and societal concerns, or shaping the infrastructures for research environments and future research trajectories.

Many of the papers are inspired by Donna Haraway's approach to understanding responsibility. Yet they do so by tracing the practices and enactment of ethics in laboratory and policy environments where interspecies relations are already articulated through the imaginative politics of the $3 R s$. The $3 R s$ initially sought to weave together good science, good care, and socially acceptable practices of laboratory animal research in ways that have been subsequently transformed. In this Introduction, we identify just three aspects of this: first, the multiple roles allocated to individuals who use and care for animals in research; second, the distribution of feelings that matter across species and spaces; and third, how these are related in the growing attention to cultures of care in animal research. Our approach highlights the value of creating new interdisciplinary conversations, which combine the concerns of laboratory animal science and welfare with insights from social science and humanities research, and generates new sites for considering the future of laboratory animal care.

\section{Newly imagined roles}

The papers collected here are populated by an extensive cast inhabiting the roles now required to enact responsibilities across the practices of laboratory animal research. Historical perspectives from Rob Kirk and Tone Druglitrø demonstrate the processes through which these roles have been imagined, organized across existing infrastructures of animal care and experimental science, and placed within the closelyrelated political landscapes of UK and Norwegian debates on animal welfare and animal rights. Beth Greenhough and Emma Roe expand understanding of the care practiced by Animal Technologists (or ATs) today. They explore how far care for animals, which is mandated by the requirements of the 3Rs, can be supplemented by an AT's emotional engagement with, and day-to-day improvements to, an animal's experience. The roles of the Named Veterinary Surgeon (NVS) and Named Animal Care and Welfare Officer (NACWO) are examined in the paper by Pru Hobson-West and Ashley Davies. Their account 
demonstrates how NVSs and NACWOs reflect on the demands placed on them to consider the complex question of animal sentience when making choices about the species of animals used. The dilemmas are not resolved through scientific consensus, but by referring to imaginaries of the public via the more relational category of social sentience. Carmen Macleod and Sarah Hartley pick up this theme of social responsibilities, mapping out some of the ways in which the obligations now networked across laboratory animal care might hypothetically be enhanced through the addition of techniques from responsible research and innovation (RRI).

These papers thus all align with perspectives in STS showing how care is material, relational, and performative (Mol 2008; Mol, Moser and Pols 2010; de la Bellacasa 2012). However, in addition, in all the papers the empirical work shows how personal capacities to care are articulated in and through complex encounters-not only with animals and infrastructures, but also with an immense range of legal requirements and regulatory guidance. These are increasingly the focus of attention in care and policy practices (Gill et al. 2017). What is striking across this set of papers is the range and diversity of roles for animal care now named in law and fleshed out in policy guidance, alongside a ubiquitous and not always accessible array of acronyms. These require some further introduction. As Druglitrø suggests in her paper, the ethical choreography of what Thompson (2013) identifies as "good science" is in part worked out through the interlocking movements of some complex bureaucratic activities.

To embark on breeding, supplying, or using laboratory animals in most parts of the world now requires significant administrative knowledge as well as specific biological skills or emotional capacities to care. To give one example from the UK: the recently revised Home Office (2014a) Guidance on the operation of the Animals (Scientific Procedures) Act 1986 (ASPA) extends to over 160 pages. The Guidance explains that you need to be working at a licensed premise, with named individuals in place responsible for veterinary care, animal welfare, information, compliance and training, and at an inspected facility, which complies with the Home Office (2014b) Code of Practice relevant for holding and using the animals located there. This Code of Practice has 220 pages, which spell out in detail the physical facilities and accommodation required for different laboratory species. You will need to apply for a personal licence, which in turn demands you have completed relevant Home Office Training Modules, themselves accredited by the Royal Society of Biology. For procedures resulting in harm equivalent to, or higher than, that caused by expert insertion of a hypodermic needle you will also need to apply for a project licence. These project licences cover programmes of research for up to five years, and can extend beyond 80 pages, explaining the purpose, place and plan of research, all experimental protocols, the numbers of animals to be used, the severity of procedures, any expected adverse effects, planned endpoints and methods of killing, and how you will consider the 3Rs throughout your work. The project licence will be reviewed by your local institutional Animal and Welfare Ethical Body (AWERB), considered by the Home Office Inspector allocated to your establishment, subject to a harm-benefit analysis, and if approved then 
implemented with the support of the range of named animal care and welfare staff associated with your laboratory and subject to regular inspection.

The roles allocated to various individuals within these processes are vital for understanding how law guides specific experimental practices and caring responsibilities, and how struggles over ethics are enmeshed in bureaucratic practices. As Ghamari-Tabrizi suggests in her response to Haraway, the "hardest case of all will be struggled over in the actual details of prohibition and license and the details of practice in the procedures" (cited in Haraway 2008, 87). These licensing procedures and their implications for local practices are often absent from laboratory ethnographies. Perhaps the complexity of this arena is as daunting to social science as to science; the latter often now having dedicated individuals or offices to deal with the licensing practices required under ASPA. In addition, there appears to be an assumption that allocating roles for responsibility is to undermine care. As Noddings warned back in 1984, institutionalizing caring according to fixed rules risks that "caring disappears and only its illusion remains" (Noddings 1984, p26).

However, the papers gathered here suggest that something else is going on. This accumulation of textual guidance and growing attention to how roles intersect to generate a culture of care is re-opening space for engaging the sociological aspects of laboratory animal care in conversation with laboratory animal scientists. Here the work of Valverde (2003) in licensing practices in other domains may be valuable. Valverde talks about the importance of administrative knowledge in enacting licensing practices (2003, 20). For Valverde "administrative knowledge" is a hybrid epistemological category, which mixes application of "common knowledge" with legal knowledge about what is permitted under law and where. Animal research today also requires the mobilization of substantial "administrative knowledge," but this is not simply about having legal knowledge or specialist scientific and animal welfare expertise. As Kirk (2017) explains, the formulation and formalization of these roles is preceded by the creation of a shared concern for the improvement of animal care. The work of UFAW in the Interwar period is emblematic in his argument: even as it exploits the existing divisions between scientific research and animal care to reduce scientific concern and political debate, it was critical in generating a "common ground" for the professionalization of animal care, and later the "common context" bringing the diverse perspectives together around humane experimental techniques that would go on to forge the 3Rs. In this way, responsibility becomes "embodied in the very epistemology and practice of science" (Kirk 2017) through these newly imagined roles from the outset.

There remain challenges in how these roles may be supported or undermined as experimental practices change and scale up. However, in seeking ways of enhancing care the papers tend to counter sociological assertions that the spatial and social divisions of labor simply separate practices of caring from the epistemic practices of science (Holmberg 2011), or that the justifications of laboratory animal research are completed, a priori, through the humanist underpinnings of cost or harm-benefit analysis. The 
complex requirements of licensing and other regulatory practices are vital constituents of everyday practices of animal research and occupy much of the working lives of individuals across laboratory animal research. They are thus a key site for observing care practices and engaging the roles, relations and responsibilities associated with them. The energy and painstaking attention that scientists, animal technologists, and others must devote to the interpretation and implementation of these regulations merits equally careful consideration from social scientists. These papers demonstrate the value of attending to these legal and licensing practices, not only as moments of instruction or instrumentalization, but also as part of the collective process of forging subject positions in animal research.

\section{Feelings that matter}

If law and licensing practices are important in the creation of responsibilities and subjectivities of laboratory animal research, they are also important in the definition of the objects of care, through the identification of "feelings that matter" (Webster 2005, cited in Hobson-West and Davies 2017). If care is simultaneously a "vital affective state, an ethical obligation and a practical labor" (de la Bellacasa 2012, 197, cited in Greenhough and Roe 2017), it makes sense to ask how and to whom these affects, obligations, and labors are directed. Whilst the previous section explored how roles are distributed through the regulatory organization of ethical obligations, here we explore how the papers draw attention to feelings that matter across laboratory animal research. Feelings that matter are allocated across species and spaces, referring at different points to the feelings of animals and animal care takers, as well as those of wider publics. There are different experimental and other practices through which these affective states are co-produced and signified. This is perhaps most evident in the foundational definition of animal sentience as the requirement for regulatory concern in Hobson-West and Davies (2017), which turns out to be co-produced by the NVS through the practices of law, animal welfare science, and wider societal sentience. The complexity of this most fundamental recognition points to the range of issues at stake elsewhere.

First, there is the co-production of "feelings that matter" through discussions over animal quality (Druglitrø 2017) and the concurrent attempts to define and ameliorate pain, stress, and distress in laboratory animals (Kirk 2017). Even today, pain research is a challenging area for the application of the $3 R$ s as efforts to understand pain often involve the infliction of pain. In the UK law prohibits pain, suffering, distress, and lasting harm that cannot be ameliorated. But there are potential asymmetries in patterns of mattering around animal pain, with different motivations for attending to bodily manifestations of animal pain (Hobson-West and Davies 2017), and different access to human resources to change practices (Greenhough and Roe 2017). Again, the 3Rs manages this tension by aligning good science with good welfare, in a move that has enabled the 3 Rs principle to travel widely. However, what also travels with this is the assumption that a painless death is de facto a "humane" one (Kirk 2017), and 
within the UK at least, death itself is not considered as a welfare issue under ASPA, even though the killing of large numbers of animals that are bred surplus to experimental requirements puts considerable stress on animal technologists (Greenhough and Roe 2017).

Secondly, the recognition of stress as a feeling that matters, which may have implications for experimental results (Druglitrø 2017; see also Kirk 2014; Davies 2012b), also has potential impacts on the organization of laboratory animal research more broadly. As laboratory animals increased in "quality" and thus in value, the importance of the care outside of experimental spaces, and thus feelings that matter, extend into wider networks of animal breeding and supply. Recognizing the importance of animal stress - for the animal, for the experiment, and for the experimenter-can be used to extend the importance of animal welfare from the point of procedure to the whole animal life course, on the basis that it doesn't matter to animals where their suffering or stress comes from. The trans-species relationality of laboratory animal research that Haraway places at the moment of encounter, reaches back in time and outwards through space, with implications for care practices and the care of caretakers. For Russell and Burch, these questions of animal welfare were thus also necessarily ones of "human sociology; for they are determined by human needs and decisions" (Russell and Burch 1959, 32, cited in Kirk 2017). The nature of these human needs and decisions may be questionable and changeable, but attending to trans-species relationality becomes more, not less, important as the lifetime experiences of animals are bought into consideration.

The final point about feelings that matter relates to the growing recognition of societal concerns around animal research, and the identification of public stakes in responsible regulation more broadly (MacLeod and Hartley 2017). This aspect is not explicit in the work of Russell and Burch. Whilst their arguments may have presupposed a shared culture, as Kirk explains, there is "no consideration whatsoever as to the view and concerns of the lay public" (Kirk 2017). There has been a subsequent shift in who is now required to consider the concerns of the public and the ways and places through which this is performed. This is a key issue in many of the papers, but most notably in that by Hobson-West and Davies. Reducing the societal "pain, suffering, distress, and lasting harm" that is a potential consequence of laboratory animal science emerges as an implicit ordering principle in decisions made by named vets and NACWOs about animal sentience. The explicit requirement to accord importance to societal concerns about animal research is increasingly embodied in law and local practices, such as lay membership of ethical review boards.

\section{Changing cultures of laboratory animal research}

The most notable recent change in the international language of animal research regulation has been the increasing attention to the development and support of local cultures of care within establishments and facilities. Yet to return to questions of culture is something of a challenge to contemporary science and technology studies. Here analytical approaches have actively moved away from "reducing events and 
actors to a given context" (Asdal 2012, 379) and the use of a category of culture, towards attending to more tangible forms of material relationality or more affective qualities of atmospheres. It is telling that none of the papers use culture as an explanatory category, except in Kirk's identification of a now obsolete shared culture of science and humanities. Yet, this growth in culture as an actor's category demands serious attention, and several of the papers begin this process. In part, this growing discussion of the culture of care within an establishment reflects the local proliferation of named roles allocated to individuals responsible for different aspects of animal welfare. To identify how far they work together to create a good culture of care requires practical attention to how these roles interact and communicate with one another and the often palpable but more incalculable atmospheres of care that they create. In part it also reflects a growing discussion about the international regulation of animal research (MacArthur Clark 2008; Turner et al. 2015), which may include harmonizing regulations across different spaces and recognizing different national relationships between societal expectations of animal research and local regulation of care. A culture of care is thus a complex relational and spatial category from the outset.

In the UK context, the recent definition of a culture of care emphasizes the character of local establishments, wider societal expectations and the interplay of local and national governance: "A good culture of care is an environment which is informed by societal expectations of respectful and humane attitudes towards animals used in research. Each establishment will have its own way of conveying its culture of care. However, all establishments are subject to similar governance and legal responsibilities under ASPA to deliver humane care" (Home Office 2015). As Greenhough and Roe explain, having a good culture of care is often about exceeding what is mandated by law. It is an ongoing process of relating, within an establishment, which needs to be cultivated and sustained. It may be enhanced or hindered by technological and organization change, but it indicates an aspiration, such that a culture of care is expected to exceed a culture of compliance.

However, this is not a universal definition or experience. It may vary across different establishments and different national contexts. As Friese and Nuyts's commentary and bibliometric analysis underscores, culture shapes the ways in which the ethical frameworks of the 3Rs are transformed as they travel. Again, a precursor to these more cultural questions of laboratory animal care can be found in the closing chapter of Russell and Burch's 1959 book. This focuses on the psychological, sociological, and organizational factors influencing the adoption of "existing knowledge to the improvement of experimentation" $(1959,164)$. The logics operating within different research, pathology, and commercial laboratories are explained with reference to the ecologies of these settings; the different incentives for replacement, reduction and refinement in animal research are found in the flows of time, cost, and effort across each system. Those aspects of individual psychology, institutional rigidity, or legal constraint that prevent communication and change are understood through the maintenance of habit, ultimately leading 
to inertia, isolation, and extinction. The languages that Russell and Burch used in 1959 have changed but the interdisciplinary challenge they identified still endures: how to understand the ways in which situations influence the application of knowledge to the improvement of experimentation and laboratory animal care?

Understanding the cultures of laboratory animal research is not only of value in considering how local establishment cultures may hinder or enact change. There are also interdependencies with the wider cultural relations between science and society. As Germain et al. conclude in their review of changing European politics of animal experimentation, what is at stake in this debate is as much about the way the "place of science in society is articulated and scrutinized [...] in the public sphere" (Germain et al. 2017, 76). A telling contrast emerges in the way a culture of care is currently being used outside of Europe. In a policy and practitioner focused paper, Klein and Bayne (2007) discuss their aspiration for "establishing a culture of care, conscience, and responsibility" in the USA. In their introduction, they quote Dr Alan C. Rosenquist, the Chair of the University of Pennsylvania Institutional Animal Care and Use Committee (IACUC), who stated, "Let's regulate ourselves or someone with a '.gov' address will do it for us" (2007, 3). They explain: "one strategy to prevent burdensome regulations is the development and implementation of a comprehensive program of animal care and use" $(2007,3)$. Here, a culture of care is counterpoised to a culture of control.

This difference in national political culture matters, not only for local practices of animal welfare, but also in the search for theoretical resources and social scientific accounts of the places in which responsibility is enacted in laboratory ethnographies. Haraway's focus on the inter-corporeality of co-presence and the capacities of local researchers as the basis for trans-species responsibilities may be effectively aligned with the local institutional structures of animal regulation in the USA, and sometimes elsewhere. However, in Europe and the UK, the cultural landscapes of care are different, in part due to the institutional dialogue that the development and implementation of national and international regulation has instigated. Kirk suggests that originally, the " 3 Rs were never intended to be institutionalized [...] they were to be internalized: embodied in the human scientist and enacted in scientific research" (2017). The papers here indicate that they are both partially institutionalized and internalized, and that there remain gaps and tensions. However, they also demonstrate how this innovation in the identification and governance of humane research has formed a rich set of resources for future institutional practices and individual conversations about animal research and animal care. These do not exhaust the conversations to be had about interspecies intra-action, affective encounter, and ethical response-ability. However, they arguably have the potential to exceed the emphasis on identifying responsibility only through animal encounters, notably through the incorporation of efforts to reduce and ultimately replace animals in this ethical formulation from the outset. 


\section{Conclusion: Sharing responsibilities for a successor science}

The papers assembled here explore how the regulation of animal research, especially in the EU but increasingly elsewhere, is both facilitated and shaped through a raft of regulations, principles, handbooks, guidelines, standards, and norms for humane laboratory animal care. The way these construct notions of respect and responsibility have been developed and adapted over time, and increasingly include new demands for responsibility to societal concerns. The contexts in which they have been developed and subsequently implemented reflect a range of values around human-animal relations, but also the other interfaces between the humanities and sciences, and the patterns of accountability between science and society. We present them here as part of our efforts to open space for collaborative research across interdisciplinary and practitioner perspectives in laboratory animal research and testing. As Ghamari-Tabrizi implies "these details, these mundane practices, are the place where the politics of successor science get worked out" (cited in Haraway 2008, 86-87).

The importance of this beyond the laboratory animal community point is underscored in Asdal's commentary, where she argues that to understand change requires "attending to how entities, collectives or assembles are modified and transformed, over time, by way of little tools, such as, for instance law, principles and rules." We welcome this growing attentiveness to the liveliness of guidelines and handbooks, cultures of care, and licensing practices, which are as central to the imaginative politics of animal research and use as trans-species relations. In responding to Asdal's questions about "interest" (2017), we are interested in how this special issue can contribute to conversations seeking to move beyond critique and explore how to enact more responsible practices in science (Thompson 2013). We also find interesting the current dialogue between histories of science and science and technology studies (Daston 2009; see also Druglitrø 2016) and how, in this case, attending to changing framings around science in the past has the potential to inform and take us beyond forms of relating in the present. Overall, we suggest that the history of the development of humane techniques in animal research demonstrate how debates about humanism, and its alternatives, are always already complexly involved in enacting situations.

Collaboration among the humanities, social sciences and sciences may further laboratory animal science and welfare, and so there is value in going back to recover the interdisciplinary basis of Russell and Burch's 1959 volume. However, this also uncovers warnings. As Kirk identifies, the book had as its origins an "earlier ethos wherein humanistic and scientific values occupied a shared culture" (Kirk 2017). Yet even at its publication, its eclectic, multi-disciplinary style limited its ability to travel, and its mix of cybernetics and psychoanalysis has "little traction today" (Kirk 2017). Whilst interdisciplinary conjunctions can be productive, speaking across disciplines and communities can be challenging (see 
Davies et al 2016). Debates in the UK, Europe and USA have focused attention more firmly on questions of experimental reproducibility and replicability, as well as animal model relevance (see for example AMS 2015; Würbel 2017; Garner et al. 2017). These are part of a greater focus on the translation of animal research into health and clinical outcomes, but they also reflect related moves towards open science and data sharing, and an increasingly critical attention to research integrity and reproducibility.

The contexts in action for laboratory animal science (Asdal 2012) thus both differ broadly and reach deeply into the practices of animal care. As Kirk puts it, "the wider social, cultural and political context surrounding the scientific use of animals should then be seen as integral to the transformation of animal care and experimental practices over time. This historical process might best be seen as the animal research nexus" (Kirk 2017). This nexus of relational historical processes in which new subject positions are imagined, feelings that matter are co-produced and cultures of care transformed connect Haraway's emphasis on sharing suffering in the laboratory with the locally situated but also extended responsibilities for animal care. We hope this collection will inspire further exploration of potential for shared responsibilities through the intersections of the experimental protocols and bureaucratic activities of laboratory animal science and welfare. Nearly 60 years after the publication of Russell and Burch, we see an urgent need for new, collaborative research that uses the humanities and social sciences to fully explore the dynamics of the animal research nexus today.

\section{Acknowledgements}

Working across disciplinary specialisms is one of the most challenging activities in academia. We are grateful for the openness, generosity, collegiality, and respect which all participants brought to the series of meetings and subsequent conversations through which our work was made possible and continues to be enriched. We gratefully acknowledge the support of the Wellcome Trust, which enabled these conversations to begin through a small grant [grant number 104339/Z/14/Z, 2014-2015] and continue in a collaborative award [205393/C/16, 2017-2022].

\section{Biographies}

Gail Davies is Professor in Human Geography at the University of Exeter. Her work is located at the intersection of human geography, science and technology studies, and animal studies. Recent research seeks to chart the changing geographies of laboratory animal research, support decision-making in complex science-policy contexts, and develop innovative public and artist engagements with science. Gail Davies was appointed to the Animals in Science Committee in 2013 and chaired the recent review of harm-benefit analysis in UK animal research.

Please see the special issue papers for biographies for Beth Greenhough, Pru Hobson-West and Rob Kirk. 


\section{References}

AMS [Academy of Medical Sciences]. 2015. Reproducibility and reliability of biomedical research: improving research practice. London, UK: The Academy of Medical Sciences. Available at https://acmedsci.ac.uk/file-download/38189-56531416e2949.pdf. Last accessed 8/11/2017

Asdal, K. 2012. "Contexts in Action-And the Future of the Past in STS." Science, Technology, \& Human Values, 37 (4): 379-403.

Birke L., Arluke A., and Michael M. 2007. The Sacrifice: How Scientific Experiments Transform Animals and People. West Lafayette, WV: Purdue University Press.

Dam, M.S. and Svendsen, M.N. 2017. "Treating pigs: Balancing standardisation and individual treatments in translational neonatology research." BioSocieties, https://doi.org/10.1057/s41292-017-0071-2.

Daston, L. 2009. “Science studies and the history of science." Critical inquiry, 35 (4): 798-813.

Davies, G. F., Greenhough, B. J., Hobson-West, P., Kirk, R. G. W., Applebee, K., Bellingan, L. C., Berdoy, M., Buller, H., Cassaday, H.J., Davies, K., Diefenbacher, D., Druglitrø, T., Escobar, M.P., Friese, C., Herrmann, K., Hinterberger, A., Jarrett, W.J., Jayne, K., Johnson, A.M., Johnson, E.R., Konold, T., Leach, M.C., Leonelli, S., Lewis, D.I, Lilley, E.J., Longridge, E.R., McLeod, C.M., Miele, M., Nelson, M.C., Ormandy, E.H., Pallett, H., Poort, L., Pound, P., Ramsden, E., Roe, E., Scalway, H., Schrader, A., Scotton, C.J., Scudamore, C.L., Smith, J.A., Whitfield, L. and Wolfensohn, S. 2016. "Developing a Collaborative Agenda for Humanities and Social Scientific Research on Laboratory Animal Science and Welfare." PLOS ONE, 11 (7), p e0158791.

Davies, G. 2012a. "What is a humanized mouse? Remaking the species and spaces of translational medicine." Body \& Society 18 (3-4): 126-155

Davies, G. 2012b. "Caring for the multiple and the multitude: Assembling animal welfare and enabling ethical critique." Environment and Planning D: Society and Space 30 (4): 623-638.

Despret, V. 2004. "The Body We Care for: Figures of Anthropo-Zoo-Genesis." Body \& Society 10 (2-3): 111-134.

Druglitrø, T. 2017. "'Skilled care" and the making of good science." Science, Technology \& Human Values, special issue volume

Friese, C. 2013. "Realizing potential in translational medicine: The uncanny emergence of care as science." Current Anthropology, 54 (S7): S129-S138. 
Garner, J.P., Gaskill, B.N., Weber, E.M., Ahloy-Dallaire, J. and Pritchett-Corning, K.R. 2017. “Introducing therioepistemology: The study of how knowledge is gained from animal research." LabAnimal 46 (4): 103-113.

Germain, P.L., Chiapperino, L. and Testa, G. 2017. "The European politics of animal experimentation: From Victorian Britain to 'Stop Vivisection'." Studies in History and Philosophy of Science Part C: Studies in History and Philosophy of Biological and Biomedical Sciences 64: 75-87.

Gill, N., Singelton, V. Waterton, C. 2017 Care and Policy Practices Sociological Review Monograph. London, UK: Sage.

Greenhough, B. and Roe, E. 2010. "From ethical principles to response-able practice." Environment and Planning D: Society and Space, 28 (1): 43-45.

Greenhough, B. and Emma R. 2017. "Exploring the role of animal technologists in implementing the 3Rs: An ethnographic investigation of the UK university sector." Science, Technology, \& Human Values, this volume

Hobson-West, P. and Davies, A. 2017. "Societal sentience: constructions of the public in animal research policy and practice." Science, Technology, \& Human Values, this volume

Holmberg, T. 2011. “Mortal love: Care practices in animal experimentation." Feminist Theory, 12 (2): $147-$ 163.

Johnson, E. 2015. "Of Lobsters, Laboratories and War: Animal Studies and the Temporality of More-thanhuman Encounters." Environment \& Planning D: Society and Space 33 (2): 296-313.

Kirk, R.G.W. 2017. "Recovering the principles of humane experimental technique: The 3Rs and the human essence of animal research." Science, Technology, \& Human Values, this volume.

Kirk, R. G. W. 2014. "The Invention of the "Stressed Animal" and the Development of a Science of Animal Welfare, 1947-86." In Stress, Shock, and Adaptation in the Twentieth Century, edited by David Cantor and Edmund Ramsden, 241-263. Rochester, NY: University of Rochester Press.

Klein, H.J. and Bayne, K.A. 2007. "Establishing a culture of care, conscience, and responsibility: Addressing the improvement of scientific discovery and animal welfare through science-based performance standards." ILAR journal, 48 (1): 3-11.

Lynch, M. 1988. "Sacrifice and the Transformation of the Animal Body into a Scientific Object: Laboratory Culture and Ritual Practice in Neuroscience." Social Studies of Science 18 (2): 265-89.

MacArthur Clark, J. 2008. "A global vision for laboratory animal medicine." ALTEX 14: 735-737. 
McLeod, C. and Hartley, S. 2017. "Responsibility and laboratory animal research governance." Science, Technology, \& Human Values, this volume.

Mol, A. 2008. The Logic of Care: Health and the problem of patient choice Abingdon, UK: Routledge

Mol, A. Moser, I. and Pols, J. 2010. "Care: Putting Practice into theory." In Care in Practice. On Tinkering in Clinics, Homes and Farms, edited by A. Mol, I. Moser and J.Pols, 7-26. Bielefeld, Germany: Transcript Verlag

Nelson, N. 2016. "Model homes for model organisms: Intersections of animal welfare and behavioral neuroscience around the environment of the laboratory mouse." BioSocieties 11 (1): 46-66.

Noddings, N. 1984. Caring: A feminist approach to ethics and moral education. Berkeley: University of Berkeley Press.

de la Bellacasa, M. 2012. "Nothing comes without its world. Thinking with care." The Sociological Review 60 (2): 197-216.

Russell, W.M.S. \& Burch, R.L. 1959. The Principles of Humane Experimental Technique. London, UK: Methuen

Thompson, C. 2013. Good science: the ethical choreography of stem cell research. Cambridge, MA: MIT Press.

Turner, P.V., Pekow, C., Clark, J.M., Vergara, P., Bayne, K., White, W.J., Kurosawa, T.M., Seok, S.H. and Baneux, P., 2015. "Roles of the international council for laboratory animal science (ICLAS) and international association of colleges of laboratory animal medicine (IACLAM) in the global organization and support of 3Rs advances in laboratory animal science." Journal of the American Association for Laboratory Animal Science 54 (2): 174-180.

Valverde, M. 2003. Law's Dream of a Common Knowledge. Princeton, NJ: Princeton University Press.

Weisberg, Z. 2009. "The broken promises of monsters: Haraway, animals and the humanist legacy." Journal for Critical Animal Studies 7 (2): 22-62.

Würbel, H. 2017. "More than 3Rs: the importance of scientific validity for harm-benefit analysis of animal research." LabAnimal 46 (4): 164. 